\title{
CRISPR/Cas9-mediated mutagenesis of CIBG1 decreased seed size and promoted seed germination in watermelon
}

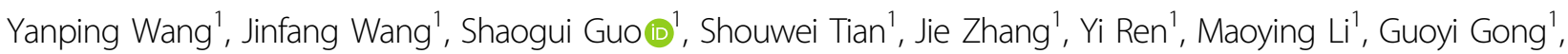 \\ Haiying Zhang ${ }^{1}$ and Yong Xu(D)
}

\begin{abstract}
Abscisic acid (ABA) is a critical regulator of seed development and germination. $\beta$-glucosidases (BGs) have been suggested to be contributors to increased ABA content because they catalyze the hydrolysis of ABA-glucose ester to release free ABA. However, whether BGs are involved in seed development is unclear. In this study, a candidate gene, CIBG1, in watermelon was selected for targeted mutagenesis via the CRISPR/Cas9 system. Seed size and weight were significantly reduced in the Clbg1-mutant watermelon lines, which was mainly attributed to decreased cell number resulting from decreased ABA levels. A transcriptome analysis showed that the expression of 1015 and 1429 unique genes was changed 10 and 18 days after pollination (DAP), respectively. Cytoskeleton- and cell cycle-related genes were enriched in the differentially expressed genes of wild type and Clbg1-mutant lines during seed development. Moreover, the expression of genes in the major signaling pathways of seed size control was also changed. In addition, seed germination was promoted in the Clbg 1-mutant lines due to decreased ABA content. These results indicate that CIBG1 may be critical for watermelon seed size regulation and germination mainly through the modulation of ABA content and thereby the transcriptional regulation of cytoskeleton-, cell cycle- and signaling-related genes. Our results lay a foundation for dissecting the molecular mechanisms of controlling watermelon seed size, a key agricultural trait of significant economic importance.
\end{abstract}

\section{Introduction}

The seed is a unique organ in a seed plant and is crucial for the plant life cycle. Seed size and weight are important agronomic traits, and in addition to influencing plant fitness and adaption to environmental stresses, seeds can also affect yield and quality, which is especially true for plants for which seeds are the main product organ ${ }^{1-3}$. The seeds of watermelon (Citrullus lanatus (Thunb.) Matsum. \& Nakai) have dual uses. Edible watermelon seeds provide humans with rich nutrition, such as oil and protein $^{4}$;

Correspondence: Yong Xu (xuyong@nercv.org)

${ }^{1}$ National Watermelon and Melon Improvement Center, Beijing Academy of Agricultural and Forestry Sciences, Key Laboratory of Biology and Genetic Improvement of Horticultural Crops (North China), Beijing Key Laboratory of Vegetable Germplasm Improvement, Beijing 100097, China

These authors contributed equally: Yanping Wang, Jinfang Wang therefore, large seeds are preferred; in contrast, for fleshconsumed watermelons, no (or small and sparse) seeds are better because the flesh portion is larger, which improves consumer experience ${ }^{1}$. Therefore, seed size is an important horticultural trait for selection during watermelon breeding. However, the molecular mechanisms underlying seed phenotype remain unclear.

Seed development is a very complex process. Seed size regulation involves numerous genes that respond to developmental and environmental signals ${ }^{5,6}$. Recent studies have revealed some key genes and regulatory pathways that control seed size in plants ${ }^{6,7}$, including $G$ protein signaling, the ubiquitin-proteasome pathway, mitogen-activated protein kinase (MAPK) signaling, some transcriptional regulators, and phytohormone perception and homeostasis. G protein signaling affects multiple

\section{(c) The Author(s) 2021}

(c) (i) Open Access This article is licensed under a Creative Commons Attribution 4.0 International License, which permits use, sharing, adaptation, distribution and reproduction in any medium or format, as long as you give appropriate credit to the original author(s) and the source, provide a link to the Creative Commons license, and indicate if changes were made. The images or other third party material in this article are included in the article's Creative Commons license, unless indicated otherwise in a credit line to the material. If material is not included in the article's Creative Commons license and your intended use is not permitted by statutory regulation or exceeds the permitted use, you will need to obtain permission directly from the copyright holder. To view a copy of this license, visit http://creativecommons.org/licenses/by/4.0/. 
aspects of physiological activities during plant growth and development. The functional $\mathrm{G}$ protein is a heterotrimeric complex composed of three subunits, namely, the $G \alpha, G \beta$, and Gy subunits. In Arabidopsis, a Gy (AGG3)-deficient mutant has small seeds ${ }^{8,9}$. In rice, GS3 and DEP1, which share significant similarity with Arabidopsis AGG3, were also found to be involved in grain size regulation ${ }^{10,11}$. Recent studies revealed that the regulation of these Gys in

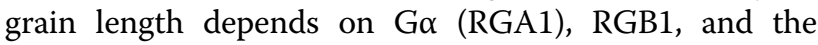
transcription factor MADS1 ${ }^{12,13}$. The ubiquitinproteasome pathway has recently been revealed to be of importance in seed size regulation. In Arabidopsis, together with the E3 ubiquitin ligases DA2 and BB/EOD1, the ubiquitin receptor DA1 was found to regulate seed growth $^{14}$. The homolog of DA1 in rice, wheat, and maize (GW2) was also reported to be involved in grain size determination $^{15-17}$. Moreover, mutation in UBP15 (ubiquitin-specific protease 15) encoded by suppressor of DA1 (SOD2) led to decreased seed and organ size. In addition, mutations in factors participating with the $26 \mathrm{~S}$ proteasome and anaphase-promoting complex/cyclosome (APC/C) ubiquitin ligases, such as SAMBA, cause large seeds ${ }^{18}$. Plant mitogen-activated protein kinase (MAPK) cascades, consisting of MAPK, MAPK kinase (MAPKK), and MAPKK kinase (MAPKKK), play important roles in a number of signal transduction pathways. In Arabidopsis, the $m k k 4 / m k k 5$ double mutant exhibited a short seed ${ }^{19}$. In rice, OsMKKK10, OsMKK4, and OsMAPK6 act tandemly to control grain size in a positive way ${ }^{20}$. In addition to the aforementioned factors, transcriptional regulators, including transcription factors, transcriptional coactivators, and chromatin modification regulators were also reported to participate in the regulation of seed size. The SQUAMOSA promoter-binding protein-like family of transcription factors, including members OsSPL13 and OsSPL16, positively affect rice grain size by modulating the expression of SRS5 and GL7, respectively ${ }^{21,22}$. OsmiR396, OsGRF4, and OsGIF cooperatively function to regulate rice grain size in a positive way ${ }^{23}$. The Arabidopsis B3 domain transcriptional repressor NGAL acts synergistically with KLU to regulate seed $\operatorname{size}^{24}$. In legumes, BS1, a plant-specific transcription regulator, was discovered to be critical for seed size determination ${ }^{25}$. Arabidopsis SMOS1 and TTG2, encoding the AP2 and WRKY transcription factors, respectively, were also found to be important in the control of seed size ${ }^{26}$.

Plant hormones have diverse functions during plant growth and development. BR and auxin are considered important regulators of seed size ${ }^{27}$. BR biosynthesis and signaling genes affect seed size ${ }^{28,29}$. Moreover, auxin biosynthesis, transport, and signaling are critical for seed size control ${ }^{30,31}$. Another important hormone, abscisic acid (ABA), is known for its prominent role in seed dormancy and germination control ${ }^{32}$. However, its role in seed size regulation has rarely been reported. The biological function of ABA depends on both its levels and signal transduction. Endogenous ABA levels are upregulated by de novo biosynthesis and/or by the one-step hydrolysis of Glc-conjugated ABA (ABA-GE), as catalyzed by $\beta$-glucosidase $(B G)^{33}$. In this study, using the CRISPR/ Cas9 system, watermelon Clbg1 mutants (mutations in $C l B G 1$, whose partial coding sequence was reported by $\mathrm{Li}$ et $\mathrm{al}^{34}{ }^{34}$ ) were generated and found to cause decreased ABA content, smaller seed size and increased germination potential. To determine the mechanisms underlying these phenomena, paraffin sections were prepared to find an explanation at the cellular level. Moreover, we performed a transcriptome analysis at two relatively early seed development stages and two time points after seed imbibition in wild-type (WT) and Clbg1-mutant lines. The expression of genes in the major signaling pathways of seed size control was also investigated. The findings expand our knowledge of the role of ABA in seed size control and may be useful for breeding seed-edible watermelon.

\section{Results \\ CRISPR/Cas9-engineered mutations in CIBG1 led to decreased $A B A$ content and seed size}

ABA is a critical factor in regulating watermelon fruit ripening ${ }^{35}$. In addition to NCEDs, which encode the ratelimiting enzyme in ABA biosynthesis, $\beta$-glucosidase also contributes to an increased level of free ABA. To isolate the putative BGs genes from the watermelon genome database, BLASTP searches of the watermelon protein database were performed based on the known BGs in tomato, grape, Arabidopsis and strawberry. Ultimately, nine $C l B G$ genes were identified in watermelon, and three of these genes were negligibly expressed (Figs. 1A and S1). Among the six expressed $C l B G$ genes in watermelon, the expression of ClBG1 (Cla97C08G153160) in cultivated watermelon 97103 increased with fruit development, while it was relatively less expressed and more stable in wild watermelon PI296341-FR (Figs. 1B and S1), which is in accordance with ABA content variation during watermelon fruit development ${ }^{35}$, making it the top candidate for involvement in releasing ABA from its conjugate form in the process of watermelon fruit ripening.

To further investigate the function of $C l B G 1$ in watermelon, we mutated ClBG1 through the CRISPR/ Cas9 system in cultivated watermelon variety ZXJM. Two sequences located in the first exon were selected as targets (Fig. 1C), which were used to obtain the desired watermelon plants with dysfunctional ClBG1. In addition, to test whether there are potential off-target sites, the two target sequences of $C l B G 1$ were searched in the watermelon genome database (http://cucurbitgenomics.org/) by BLAST with a low $E$-value $\left(1 e^{-1}\right)$. Except for the target 
A

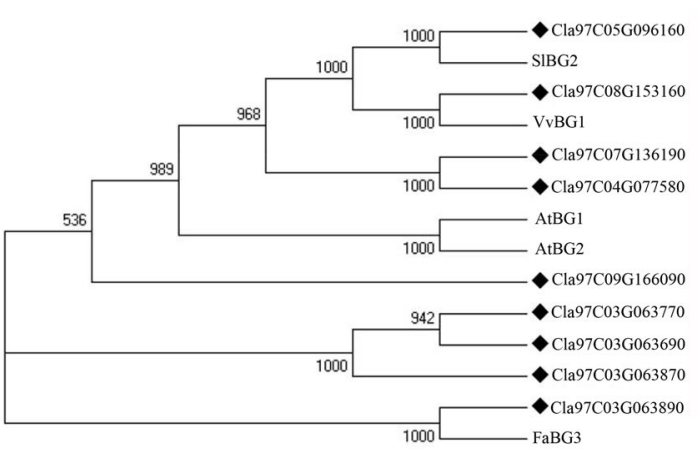

$\mathrm{C}$

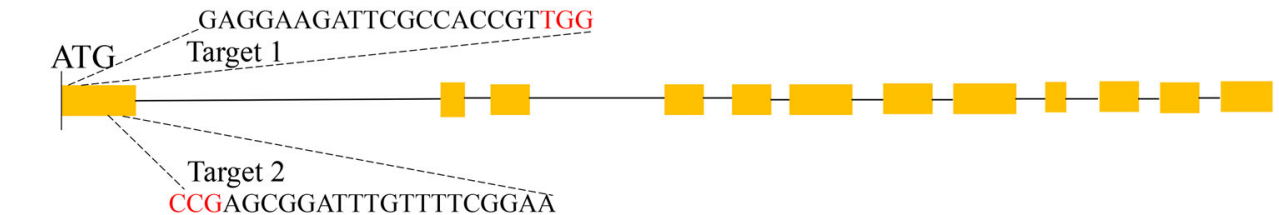

$\mathrm{D}$

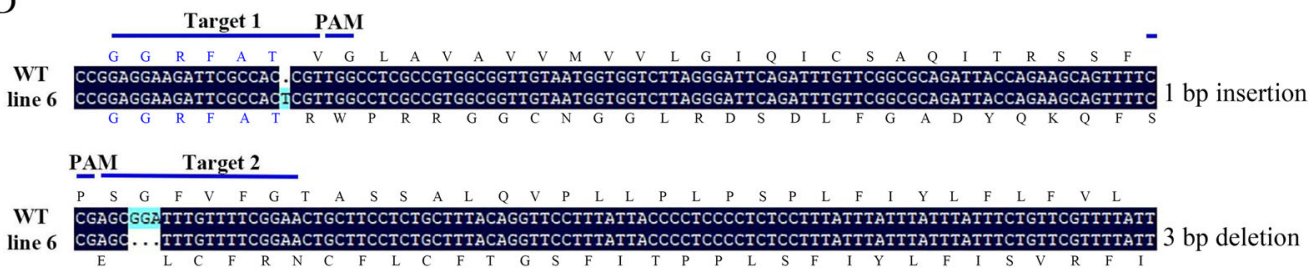

Target 1 PAM

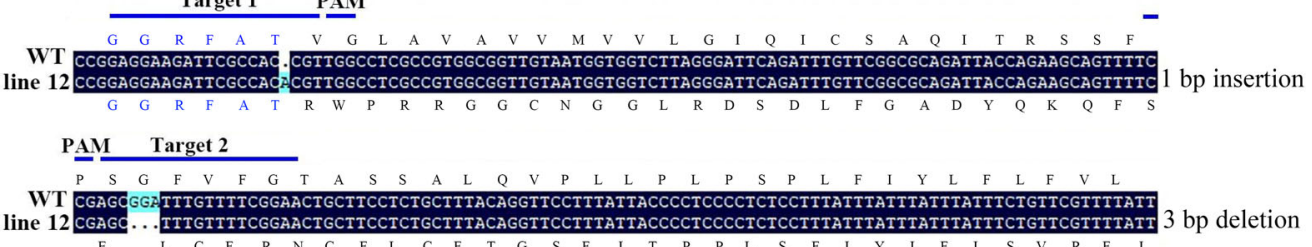

Fig. 1 A Phylogenetic trees of the BG gene family. Genes from watermelon are labeled with black diamonds. The phylogenetic trees are shown only in the form of topology structures. Sequence data from other species can be found in the GenBank data libraries under the following accession numbers: tomato-SIBG2 (Solyc07 g063880), grape-VvBG1 (XM_002274626), Arabidopsis-AtBG1 (841670) and Arabidopsis-AtBG2 (824893), and strawberry-FaBG3 (JX244263). B Expression of CIBG1 (Cla97C08G153160) in the 97103 and PI296341-FR lines during watermelon fruit development. C Schematic diagram of CRISPR/Cas9-targeted sites in the CIBG1 gene. Orange boxes represent exons; lines represent introns. PAMs (NGGs) are shown in red, and the sequence of each gRNA is shown. D Mutation sites in the Clbg1 homozygous mutant lines from the T4 generation. Frameshift mutations for encoding sequences are also shown

sites, no additional sites were found in the watermelon genome, indicating that off-target events would be rare upon ClBG1 mutation. As a result, 40 basta-resistant watermelon lines were obtained from the T0 generation, and Sanger sequencing analyses revealed that almost all these lines had the expected mutations at the targeted sites. To obtain homozygous Clbg1 mutants without Cas9, a total of $60 \mathrm{~T} 2$ plants were detected, and two types of ClBG1 mutations without Cas9 (first target, 1-bp insertion; second target, three-bp deletions) were obtained. These mutations in the first exon resulted in frame shifts (Fig. 1D)

As expected, the ABA content in Clbg1-mutant plant lines was significantly decreased (Fig. S2). Surprisingly, the process of fruit development and ripening was not significantly altered in the Clbg1-mutant lines compared to that in the WT line (Fig S3), while seed size was significantly decreased, including decreased seed weight, length, and width (Fig. 2). Therefore, more attention was given to the effect of $C l B G 1$ on watermelon seed development. To reveal the mechanisms of seed size variation in Clbg1-mutant lines at the cellular level, mature seeds were sectioned. The cell number was significantly decreased in the Clbg1-mutant lines compared to the WT line, while the cell size was significantly enlarged (Fig. $3 \mathrm{~A}-\mathrm{C})$. In addition, the $\mathrm{ABA}$ content in seeds was detected. Our results showed that ABA was increased from 10 DAP to 18 DAP in the WT plants, which was 


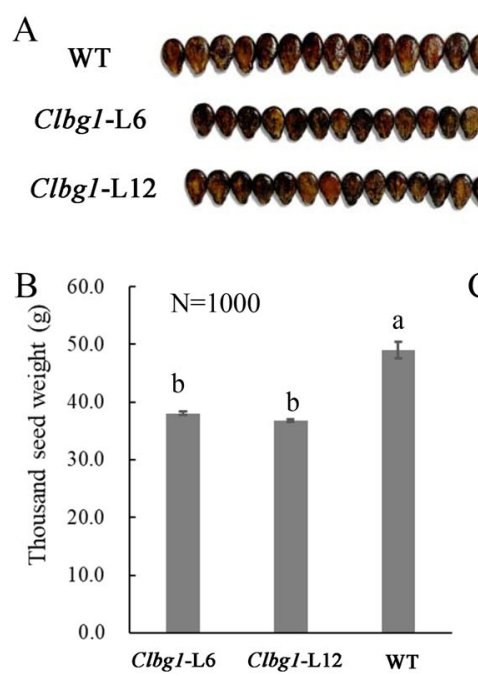

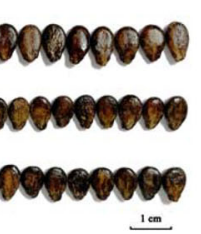

icm

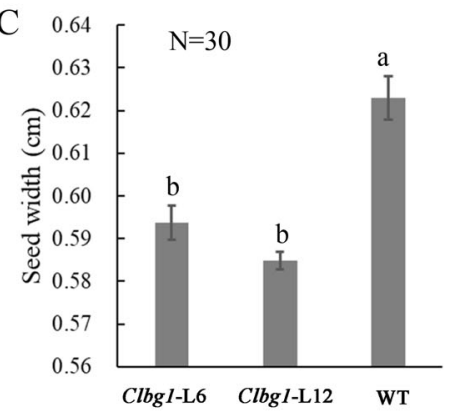

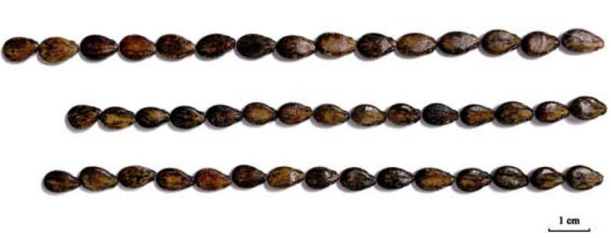

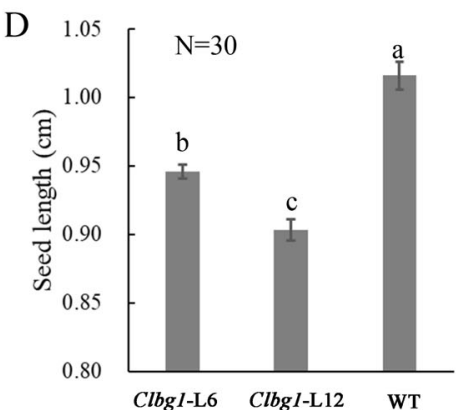

Fig. 2 Seed size variation in the Clbg1-mutant lines. Seed phenotype (A); weight of one thousand seeds (B); average seed width (C) and length (D); $N$ stands for the number of seeds that were analyzed for each genotype and each replication. Standard error is indicated. Different letters represent significant differences as determined using ANOVA followed by Tukey's HSD test $(p<0.05)$

consistent with our previous study ${ }^{35}$. However, in the Clbg1-mutant lines 6 and 12, the ABA content was lower at 18 DAP than at 10 DAP (Fig. 3D). As expected, the ABA content was significantly decreased in seeds of Clbg1 mutants compared the WT plants at both 10 and 18 DAP (Fig. 3D).

\section{Cytoskeleton- and cell cycle-related genes were involved in watermelon seed size regulation}

To understand the reason(s) that seed size was decreased in the Clbg1 mutants at the transcription level, a comparative transcriptome analysis of the wild type and Clbg1-mutant line was performed during two key seed developmental stages. Ten days after pollination (DAP), 1200 differentially expressed genes (DEGs) were identified, with 775 downregulated and 445 upregulated in the Clbg1-mutant lines (Fig. 4A). Moreover, 18 DAP, 1614 genes were significantly changed in the Clbg1-mutant lines, of which 796 genes were downregulated and 818 genes were upregulated (Fig. 4B). When these DEGs were compared, the expression of 185 genes was significantly changed at both 10 and 18 DAP, while the expression of 1015 and 1429 genes was specifically changed 10 and 18 DAP, respectively (Fig. 4C). To further understand the function of these DEGs, GO annotation analysis was performed (Fig S4). Both 10 DAP and 18 DAP, genes in metabolic processes were the most highly represented in the biological process category (540/644 genes 10 and 18 DAP, respectively), suggesting that certain important metabolic activities during seed development were affected in the Clbg1-mutant lines; genes associated with the membrane (382 genes $10 \mathrm{DAP} / 492$ genes $18 \mathrm{DAP}$ ) were the most enriched in the cellular component category; and catalytic activity-related genes (478 genes 10 DAP and 635 genes $18 \mathrm{DAP}$ ) were prominently represented in the category of molecular function (Fig S4A, B). Moreover, a $\mathrm{GO}$ enrichment analysis was also conducted for these DEGs (Fig. 5). The top 16 enriched GO terms 10 and 18 DAP are shown in Fig. 5A, B, respectively. Ten days after pollination, "biological regulation", "regulation of metabolic process", "regulation of macromolecule metabolic process", "regulation of cellular metabolic process" and "regulation of nitrogen compound metabolic process" were the top five GO terms associated with gene enrichment, in descending order. Eighteen days after pollination, "microtubule-based process", "cytoskeletal protein binding", "microtubule binding", "tubulin binding" and "cell cycle process" were the GO terms ranked among the top five with the most genes. Tubulin family proteins are the main constituents of microtubules in living cells, and microtubules are among the vital components that make up the cytoskeleton. Overall, cytoskeleton-related genes were enriched in the DEGs in the Clbg1-mutant line 18 DAP. In addition, genes involved in mitotic cell cycle processes and motor activity were also highly enriched. Taken together, the GO analysis results lay the foundation for further investigating specific pathways that are affected in the Clbg1-mutant lines during seed development and ultimately lead to smaller seeds.

In addition, to gain more insight into seed size variation in the Clbg1-mutant lines, the expression of homologous watermelon genes in the recently identified signaling pathways that control seed size was also analyzed in both the Clbg1-mutant and WT lines 10 and 18 DAP. In the 

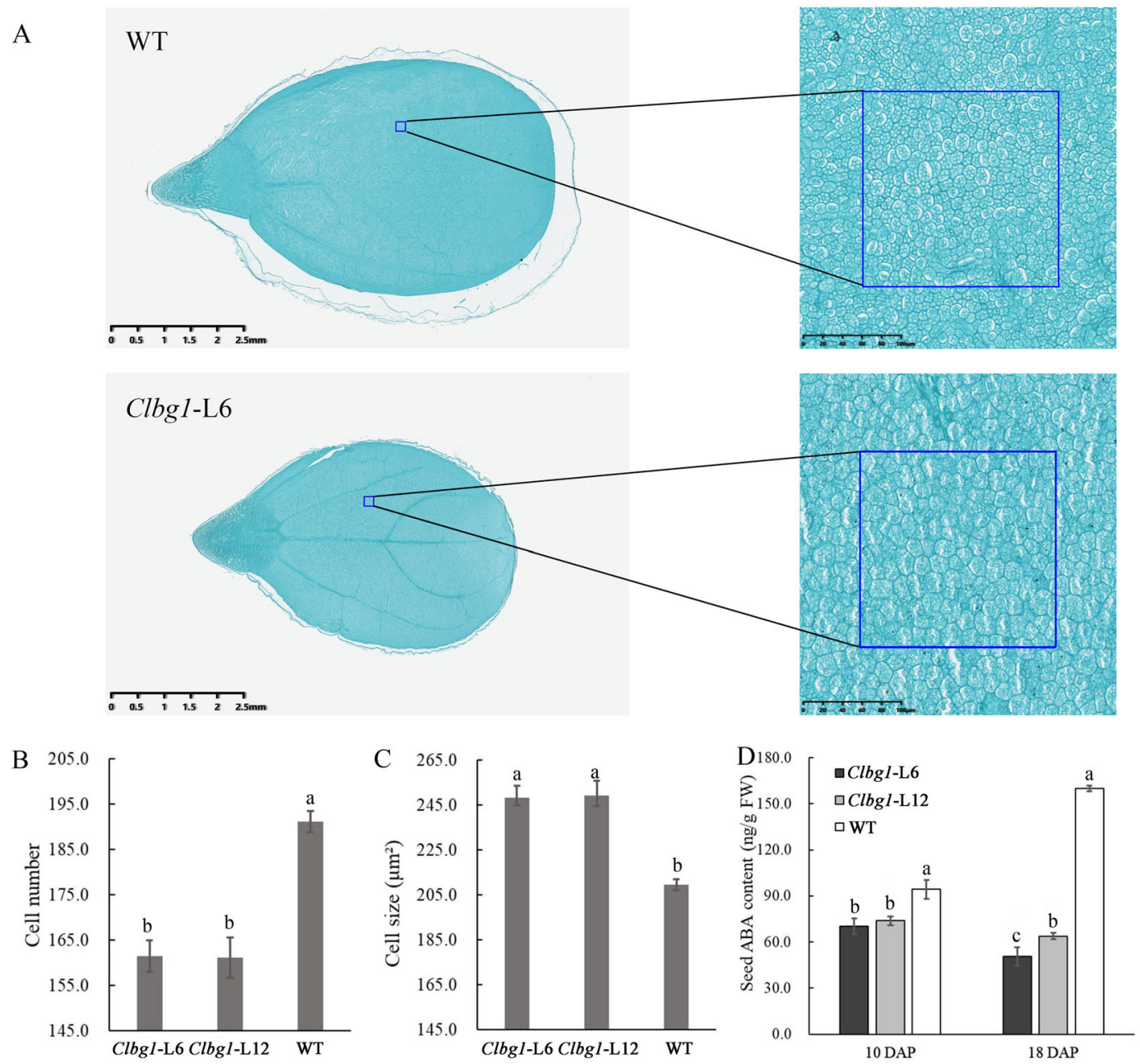

Fig. 3 The Clbg1 mutation affected seed size by decreasing cell number. Paraffin sections of mature WT and Clbg1-mutant seeds cut in the longitudinal direction (A); cell number (B) and cell size $(\mathbf{C})$ in the mature seeds of the Clbg 1 mutants and WT plants; ABA content in the seeds 10 and 18 DAP (D); standard error is indicated. Different letters represent significant differences as determined using ANOVA followed by Tukey's HSD test $(p<0.05)$

ubiquitin-proteasome pathway, only the expression of Cla97C09G166490 (EOD1) and Cla97C05G096960 (SOD2) was significantly changed in the Clbg1-L6 mutant line, compared to that in the WT line, as shown in Fig. 6. The expression of Cla97C09G166490 (EOD1) was significantly higher in the Clbg1-L6 mutant line than in the WT line 10 DAP, consistent with its negative role in regulating seed size in Arabidopsis $^{14}$. In addition, Cla97C05G096960 (SOD2) was significantly downregulated in the Clbg1-L6 mutant line $18 \mathrm{DAP}$, which may contribute to the small seed size. In $\mathrm{G}$ protein signaling, the expression levels of Cla97C11G207780 (AGG3) and Cla97C06G116080 (RGG1) were relatively low in both the Clbg1-L6 mutant and WT lines. Among the four highly expressed genes Cla97C05G096780 (GPA1), Cla97C02G032020 (AGB1), Cla97C08G158740 (RGB1) and Cla97C06G111440
(MADS1), only Cla97C08G158740 (RGB1) was differentially expressed between the Clbg1-mutant and WT lines. Cla97C08G158740 (RGB1) had significantly low expression in the Clbg1-mutant line 18 DAP. Based on its positive effect on seed size regulation, low Cla97C08G158740 expression may lead to smaller seed size. In the mitogenactivated protein kinase (MAPK) signaling pathway, Cla97C07G141020 (MKK4/5) and Cla97C01G004750 (MKKK10) showed no differential expression between the Clbg1-L6 mutant and WT lines. Cla97C03G053010 (MAPK6) was significantly downregulated in the Clbg1-L6 mutant line, which may have resulted in a smaller seed size. Although Cla97C07G131390 (MAP) was differentially expressed between the Clbg1-L6 mutant and WT lines 18 DAP, it may be less important due to its relatively low expression. Three genes in the auxin pathway, namely, 

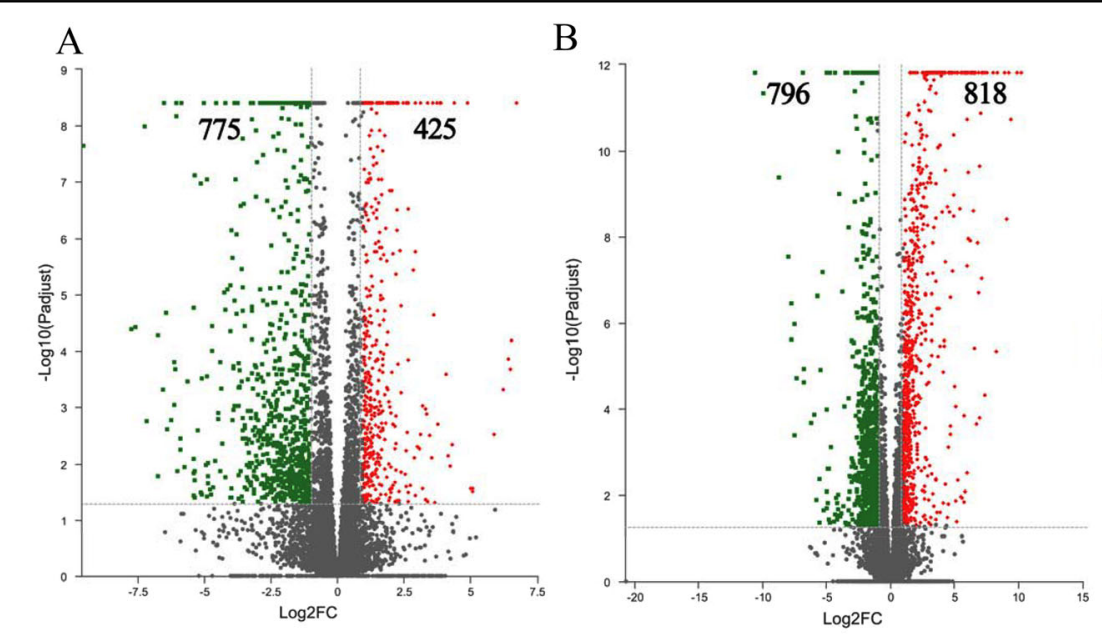

C

WT VS Clbgl-L6 (10 DAP) WT VS Clbgl-L6 (18 DAP)

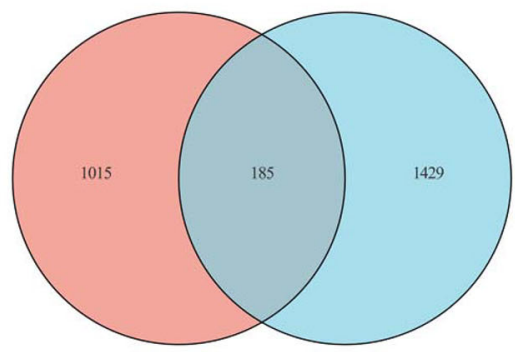

Fig. 4 DEGs analysis 10 and 18 DAP. A, B Volcano plot showing differences in the WT and Clbg1-mutant lines 10 and 18 DAP, respectively. Green indicates the downregulated genes, and red represents the upregulated genes. $\mathbf{C}$ Venn diagram showing the number of specific and common DEGs 10 and 18 DAP

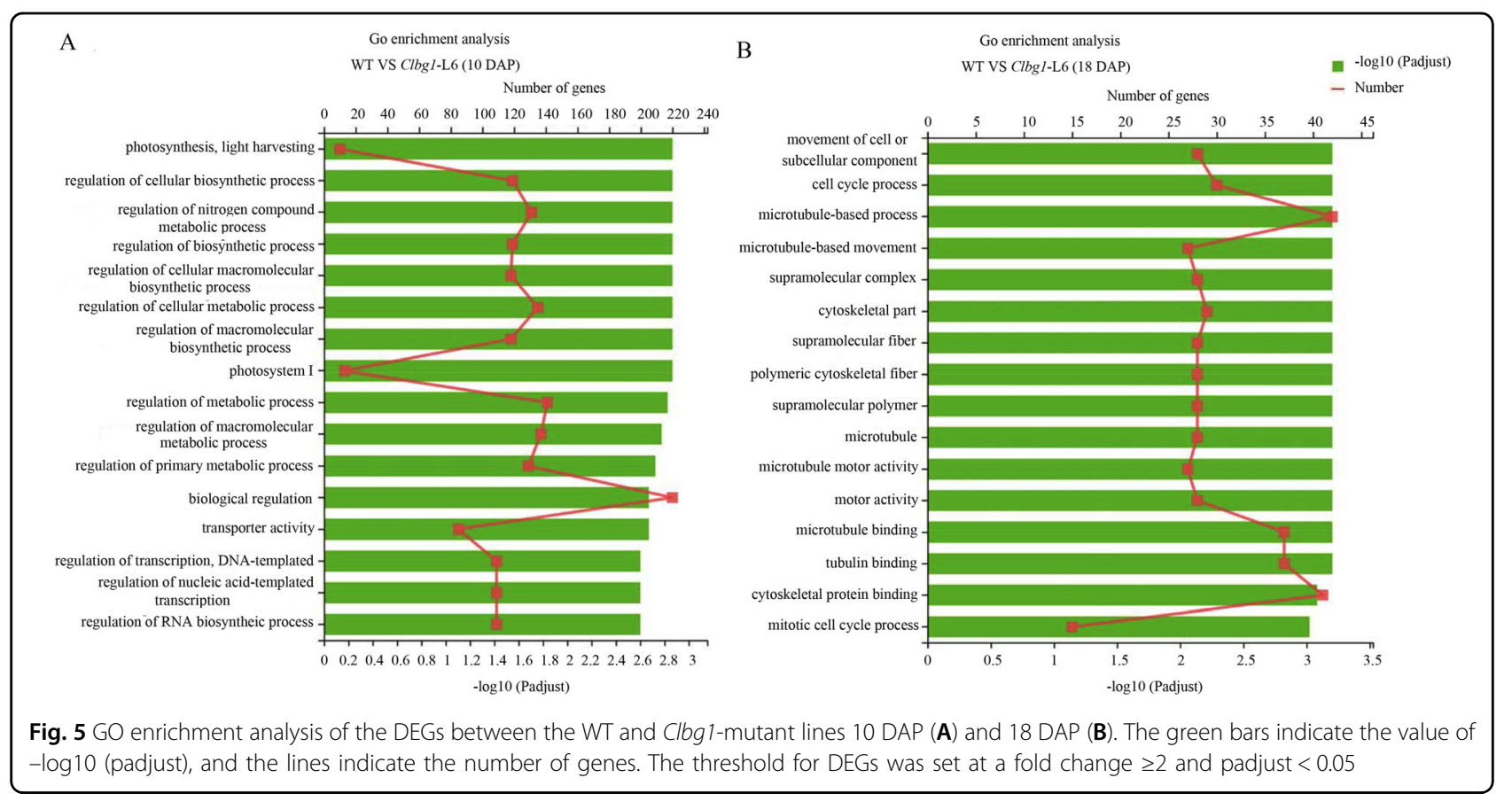

Cla97C07G140760 (BG1), Cla97C06G128110 (ARF2/4), and Cla97C04G073770 (SK41), showed no differential expression between the Clbg1-L6 mutant and WT lines. In the brassinosteroid pathway, only Cla97C01G014900 (BRI1), Cla97C06G121690 (GS5), and Cla97C03G066390 (GW5/GSE5) were differentially expressed between the Clbg1-L6 mutant and WT lines. The expression of Cla97C01G014900 (BRI1) was significantly decreased in the Clbg1-L6 mutant line 18 DAP, indicating that it may play a role in reducing seed size. Cla97C06G121690 (GS5) was significantly downregulated in the Clbg1-L6 mutant line compared to that in the WT line; however, its role in regulating seed size at the transcriptional level may be less profound because of its relatively low expression. Moreover, the expression of the negative seed size regulator GW5/ GSE5 (Cla97C03G066390) was downregulated in the Clbg1L6 mutant line $18 \mathrm{DAP}$, which is inconsistent with its role in regulating seed size in rice ${ }^{36}$. Among the 15 transcriptional regulatory factors, Cla97C06G112180 (EOD3), Cla97C08G153350 (KLU), Cla97C02G033980 (SRS5), Cla97C04G078560 (GL7), and Cla97C02G042620 (GIF) were differentially expressed between the Clbg1-L6 mutant 


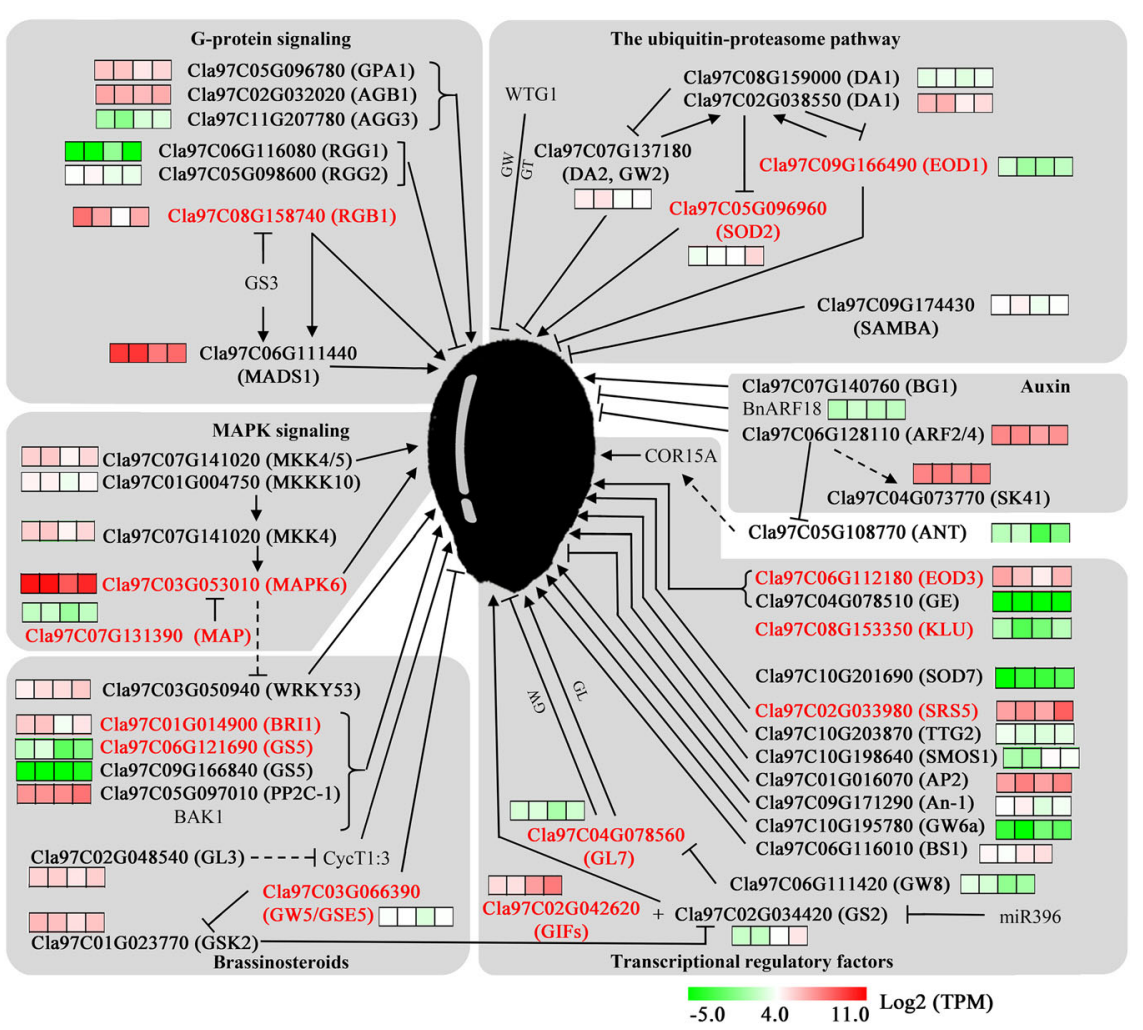

Fig. 6 The major signaling pathways of seed size control according to Li et al. ${ }^{7}$. Dashed lines represent unclear genetic relationships. Seed size regulators in Arabidopsis, rice, and other species are shown in parentheses. The heat map near each gene shows the log2 (TPM) values in the Clbg 1-L6 line and the WT line 10 and 18 DAP. The genes that were probably involved in watermelon seed size control are marked in red

and WT lines, and all of these genes were downregulated in the Clbg1-L6 mutant line 18 DAP, consistent with their positive role in regulating seed size.

\section{Clbg1 mutation promoted seed germination and altered the expression of associated genes}

To investigate the effect of $C l B G 1$ on seed germination, the germination rate was recorded periodically after seed imbibition. Both the Clbg1-L6 and Clbg1-L12 mutant lines exhibited very similar germination potential, which was higher than that in WT during the germination process (Fig. 7A). Twenty-four hours after imbibition (HAI), both the Clbg1-L6 and Clbg1-L12 mutant lines reached a germination rate of approximately $33.3 \%$, while this rate was only $5.3 \%$ in the WT line. The Clbg1-mutant lines had completely germinated 28 HAI; however, it took another 8 hours for the WT seeds to completely germinate. The ABA content variation was significantly decreased 24 HAI compared to that at $4 \mathrm{HAI}$ in both the Clbg1-mutant and the WT lines. Moreover, there was an obvious decrease in ABA content in the Clbg1-mutant lines compared to that in WT 4 HAI (Fig. 7B). Therefore, seed germination occurred earlier and the germination rate was higher in the Clbg1-mutant lines. Twenty-four hours after imbibition, the ABA content was similar in the Clbg1-mutant and WT lines, which was in accordance with the similar seed germination rate $24 \mathrm{HAI}$ (Fig. 7A, B). Although the seed germination potential of the Clbg1mutant lines was increased, radicle elongation seemed to be have been delayed (Fig S5).

To clarify the increased seed germination potential in Clbg1-mutant lines at the transcriptional level, the expression of certain seed germination indicator genes and ABA signaling pathway genes was analyzed. The expression of seed germination repressors, such as Cla97 C10G202040 (ABI5), was significantly decreased in the Clbg1-L6 mutant line compared to the WT line at both 4 and 24 HAI (Fig. 7C). The expression of Cla97C02G036380 (BZR1), which negatively mediates ABA signaling to promote seed germination, was significantly increased 24 HAI in the Clbg1L6 mutant line (Fig. 7D). In the ABA signaling pathway, there were 11 putative $C l P Y L$ genes, 5 putative group A ClPP2C genes, and 8 putative subclass III ClSnRK2 genes (Fig. 8). Among the ClPYL genes, most were not expressed significantly differently in the Clbg1-L6 mutant and WT lines. The expression of Cla97C01G000570 (PYL), Cla97C11G212910 (PYL), and Cla97C05G099080 (PYL) was significantly downregulated in the Clbg1-L6 mutant 


\section{A}

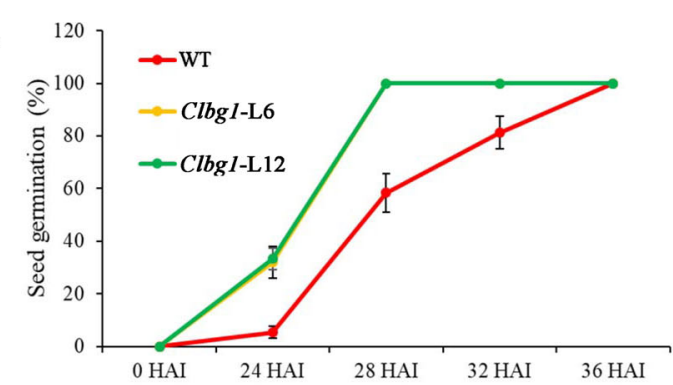

C

Cla97C10G202040 (ABI5)

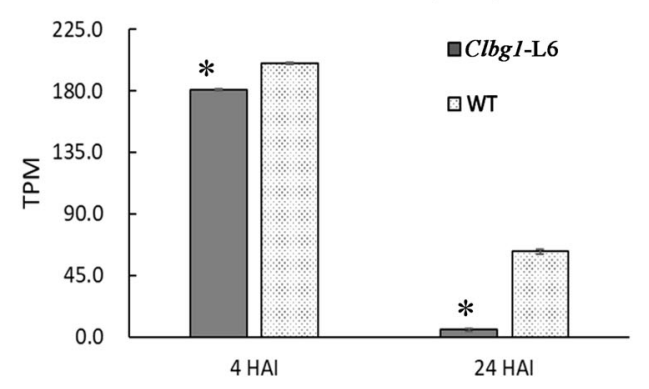

B

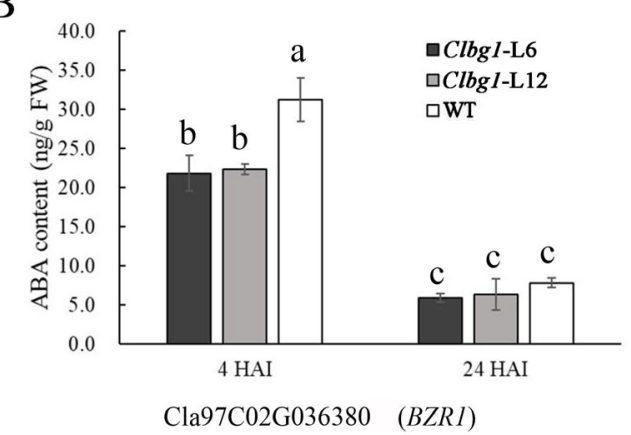

D

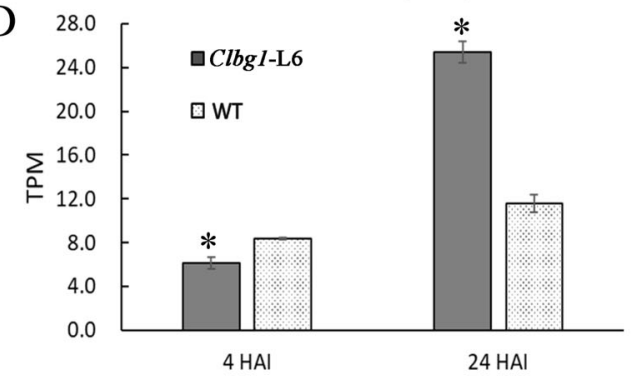

Fig. 7 Variations in seed germination rate, ABA content, and gene expression after seed imbibition. A Variation in seed germination rate in the Clbg1-L6, Clbg1-L12, and wild-type lines. B Variation in ABA content in the Clbg 1-L6, Clbg1-L12 and wild-type lines 4 and 24 HAl. C, D Expression of the ABI5 and BZR1 in Clbg1-L6 and WT lines 4 and $24 \mathrm{HAl}$. Standard error is indicated. Different letters represent significant differences as determined using ANOVA followed by Tukey's HSD test $(p<0.05) .{ }^{*}$ Indicates expression values that are significantly different at the 0.05 level compared to those of the WT line

line 24 HAI, while Cla97C10G205730 (PYL) was significantly upregulated. Among the five group A ClPP2C genes, the expression of four genes, namely, Cla97C05G089520, Cla97C11G213640, Cla97C03G052090, and Cla97C07G140660, but not that of Cla97C08G152820 (PP2C), was significantly decreased in the Clbg1-L6 mutant line compared the WT line. As for the ClSnRK2 genes, Cla97C09G178950 and Cla97C04G069570 were not expressed in any of the samples examined. Except for Cla97C04G070050 and Cla97C10G186750, which showed significant expression differences between the WT and Clbg1-L6 mutant lines, ClSnRK2 genes were not differentially expressed.

\section{Discussion}

ABA, a classic multifaceted phytohormone, regulates many life processes of plants, including seed germination and dormancy, fruit development and ripening, and stress adaption $^{37}$. During these processes, ABA function depends on the variation of its level, which is mainly controlled by de novo biosynthesis, hydroxylation, and conjugation. The hydrolysis of ABA-glucose ester mediated by $\beta$-glucosidase, which releases active ABA, is thought to be faster in increasing ABA levels than the de novo biosynthesis pathway ${ }^{33}$ and is considered to play a key role in the regulation of endogenous ABA content in response to abiotic stresses and thus influences fruit physiology ${ }^{38-40}$. The mechanism of ABA glucosylation effects on ABA concentration, which influences seed morphology, has been rarely addressed. In this study, we found that ClBG1 (Cla97C08G153160) was highly expressed during cultivated watermelon ripening but expressed at low levels in nonripening wild watermelon PI296341-FR (Fig. 1B), suggesting that this gene may be involved in ABA-mediated watermelon fruit ripening. The development of various genome-editing systems has greatly facilitated studies on specific gene functions because they can be used to disrupt genes via frameshift mutations, including the creation of premature stop codons, and the production of different proteins ${ }^{41}$. Taking advantage of the CRISPR/Cas9 system, we abrogated the function of ClBG1 in watermelon; however, no obvious changes in the fruit ripening phenotype were observed, which may be due to the redundancy of gene family members. In an unexpected finding, the seed size was significantly decreased in the Clbg1-mutant lines and seed germination was promoted due to decreased ABA content (Figs. 2, 3D, and 7A). As shown in Fig S6, no significant differences were found between the WT and Clbg1mutant lines in the expression of ClNCED or ClCYP707A genes during seed development. These results suggest that the decrease in ABA content in Clbg1-mutated seeds was not caused by the de novo biosynthesis or degradation of ABA but by the dysfunction of ClBG1 due to the 


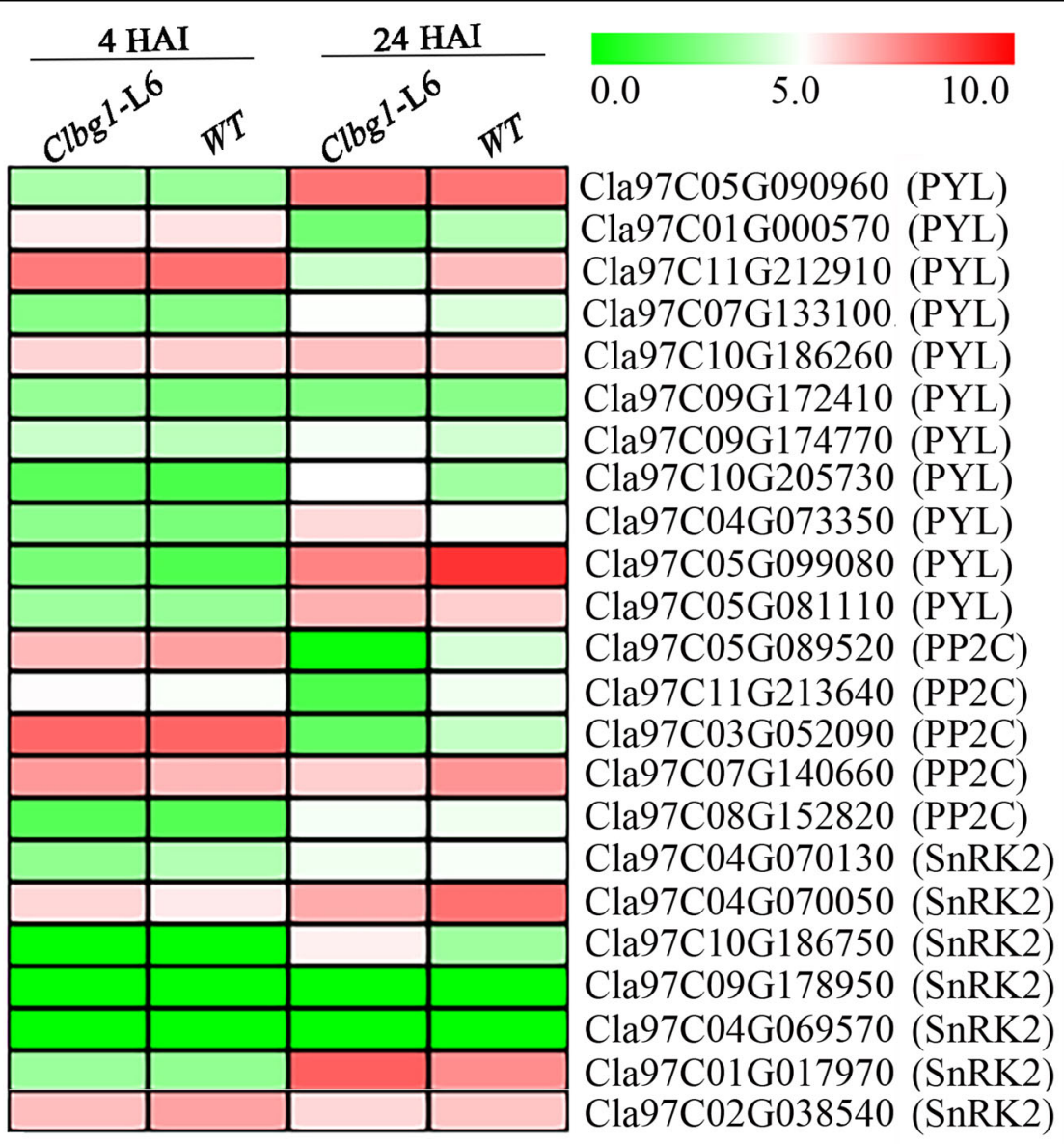

Fig. 8 Expression of ABA signaling genes in the Clbg1-L6 and WT lines $\mathbf{4}$ and $\mathbf{2 4}$ HAI. The expression values of each gene are represented as $\log 2($ TPM)

mutation, thereby decreasing watermelon seed size and promoting seed germination.

Seed size is an important agronomic trait in watermelon breeding. Several studies have identified QTLs related to seed size and weight in watermelon, mainly throughout chromosomes $1,2,3,5,6,8$, and 11 (refs. ${ }^{42-45}$ ). In this study, we found that dysfunction of $C l B G 1$ led to decreased seed size and weight. Notably, ClBG1, located on chromosome 8 (21432735-21439034, watermelon 97103 V2), was physically near to the seed size QTL CISS8.2 identified by Ren et al. ${ }^{43}$ and Prothro et $\mathrm{al}^{42}$. This finding confirms that ClBG1 may play a role in watermelon seed size control. In addition, the shape and size of plant organs are controlled by programmed cell division and cell expansion. Specifically, the spatiotemporal patterns of cell division and cell enlargement are mainly regulated by the reorientation of microtubule arrays $^{46,47}$. In this study, the GO term enrichment analysis of the DEGs revealed that microtubule-, tubulin- and cell cyclerelated genes were strongly affected by mutation in the Clbg1 gene, which was consistent with the alteration of cell number and size (Figs. 3, 5). Although the cell size was significantly increased, seed size and weight were decreased in the Clbg1- mutant lines, which indicates that cell number is the most important factor in seed size determination. In the major signaling pathways of seed size control, the function of some genes is conserved among different species. For example, in the MAPK signaling pathway, the OsMKKK10-OsMKK4OsMAPK6 cascade controls rice grain size, in which OsMKK4 and OsMAPK6 can be sequentially phosphorylated and activated by OsMKKK10, and the expression of OsMAPK6 is positively associated with cell proliferation in the spikelet hull $^{20}$. In watermelon, the expression of Cla97C01G004750 (MKKK10), Cla97C07G141020 (MKK4), and Cla97C03G053010 (MAPK6) was also positively correlated with seed size (Fig. 6). On the other hand, among the brassinosteroid-related genes, the downregulation of GSE5/ GW5 in rice led to large grains because of extensive cell proliferation in the spikelet hull ${ }^{36}$. However, downregulation of these genes had the opposite effect in watermelon. The expression of Cla97C03G066390 (GSE5/GW5) was low in the Clbg1-mutant lines with small seeds. Therefore, it can be presumed that GSE5/GW5 in rice and watermelon might have different cofactors, which leads to the opposite effects on seed size. Further investigations are needed to discover 
the different roles of genes in seed size control in different species.

\section{Materials and methods Plant materials}

The watermelon inbred line ZXJM was used in the transformation experiments mediated by Agrobacterium tumefaciens strain GV3101. ZXJM is a cultivated watermelon with red flesh and medium seed size. PI296341-FR is a nonripening wild-type watermelon variety that has white flesh and medium seed size. Flowers were artificially pollinated and labeled on the day of flowering. PI296341FR, ClBG1-edited and wild-type (WT) plants (plants derived from transformation but with no genome editing and free of Cas9) were grown under standard greenhouse conditions in the Beijing Vegetable Research Center (BVRC). The seeds were periodically harvested.

\section{Phylogenetic analysis of BGs}

The amino acid sequences of the ClBG proteins were aligned with the relatively well-studied BGs in other species, such as SlBG2 in tomato, VvBG1 in grape, AtBG1 and AtBG2 in Arabidopsis, and FaBG3 in strawberry, using ClustalX 2.0.12 software with default settings. Phylogenetic trees were constructed with MEGA 4.0.2 software using the neighbor-joining (N-J) method, and the reliability of the different phylogenetic groups was evaluated by bootstrap analysis with 1000 replicates. Tree files were viewed and edited with MEGA 4.0.2 software.

\section{Vector construction and Agrobacterium-mediated transformation in watermelon}

The binary CRISPR/Cas9 vector pBSE401 was provided by Q.J. Chen at China Agricultural University, and the vector was constructed as previously described by Xing et $\mathrm{al}^{48}$. Specific single guide RNAs (sgRNAs) targeted to ClBG1 were selected according to the assessment with CRISPR-P (http://cbi.hzau.edu.cn/crispr/). The target sequence cloned into the pBSE401 vector was named pBSE401-ClBG1, which was used to transform the watermelon cultivar ZXJM by the Agrobacteriummediated transformation method as previously described $^{49}$. The transgenic watermelon lines were selected based on basta resistance. The primers used for vector construction are listed in Supplementary Table S1.

\section{Genomic DNA extraction and mutation detection}

Genomic DNA was extracted from young leaves of T0T4 transgenic plants, which was then used for creating templates to amplify the specific fragments in the ClBG1 gene using primers flanking two targeted sites (Supplementary Table S1). PCR was conducted under the following conditions: $94{ }^{\circ} \mathrm{C} / 5 \mathrm{~min} ; 94^{\circ} \mathrm{C} / 30 \mathrm{~s}, 56{ }^{\circ} \mathrm{C} / 30 \mathrm{~s}$, and $72{ }^{\circ} \mathrm{C} / 1 \mathrm{~min}$ ( 35 cycles); and $72^{\circ} \mathrm{C} / 10 \mathrm{~min}$ as the final extension. PCR products were directly sequenced using the Sanger method by Tianyi Huiyuan Biotech Company (Beijing, China). The transgenic plants were also verified as Cas9-free with primers specific for Cas9 (Supplementary Table S1). PCR was conducted under the following conditions: $94{ }^{\circ} \mathrm{C} / 5 \mathrm{~min} ; 94{ }^{\circ} \mathrm{C} / 30 \mathrm{~s}, 60^{\circ} \mathrm{C} / 30 \mathrm{~s}$, and $72{ }^{\circ} \mathrm{C} /$ $1 \mathrm{~min}$ (29 cycles); and $72^{\circ} \mathrm{C} / 10 \mathrm{~min}$ as the final extension.

\section{Measurement of seed size and weight}

Seeds from fully mature watermelons were collected and dried. Seeds from each watermelon were randomly selected and photographed. Maximum seed length and width were measured with ImageJ software (http:// rsbweb.nih.gov/ij/). Seed weight was measured using an electronic balance. The seed weight data of 6 watermelons were combined according to plant line. This seed weight is expressed as the average of these 6 measurements for each watermelon line.

\section{Histological analysis of mature seeds}

Mature seeds of the wild-type and Clbg1-mutant lines were collected. Paraffin-embedded sections of these mature seeds were obtained by cutting in the longitudinal direction according to the method described by Dai et al. ${ }^{50}$. Images were obtained using an automatic digital pathology slide scanner (KF-PRO-120, Jiangfeng Biological Information Technology Co., Ltd, Ningbo, China) and viewed with software K-Viewer (http://www.kfbio.cn/ download.php). Cells were counted in areas of the same size $\left(40,000 \mu \mathrm{m}^{2}\right)$, as indicated in the boxes in Fig. 3, and the boxed area divided by cell number was the cell size. For wild-type and ClBG1-mutant line 6, at least three seeds were sectioned, and three sections of each seed were analyzed.

\section{Seed germination experiments}

Watermelon seeds of the wild-type and ClBG1-mutant lines with uniform plumpness were first soaked in sterilized water for 4 hours in a beaker, and then some seeds were collected as samples 4 hours after imbibition (HAI). The remaining seeds were placed into petri dishes, which were lined with moist seed-germinating paper. The germination rate (number of germinated seeds/number of total seeds) was recorded 24, 28, 32, and 36 HAI. At least 100 seeds were used for each genotype. The seed samples were immediately collected and frozen in liquid nitrogen $4 \mathrm{HAI}$ and $24 \mathrm{HAI}$ and stored at $-80^{\circ} \mathrm{C}$ until use.

\section{Determination of ABA content}

Determination of ABA content was performed using a ESI-HPLC-MS system according to the method described in our previous study ${ }^{35}$. The ABA standard $(( \pm)$-abscisic acid, A1049, Sigma, St Louis, MO, USA) was used to determine the retention time and mass spectrometric 
information of ABA. Three biological replicates of each sample were analyzed.

\section{RNA library construction, sequencing, and analysis}

RNA was isolated from lyophilized tissues with a plant RNA purification reagent kit (Invitrogen, USA). The concentration, quality, and purity of the RNA were detected with an Agilent 2100 Bioanalyzer RNA 6000 Nano kit (Agilent, USA). Twenty-four RNA libraries (seeds of the wild-type and Clbg1-L6 lines 10 DAP, 18 DAP, $4 \mathrm{HAI}$, and $24 \mathrm{HAI}$, each with three replicates) were generated with a TruSeqTM RNA Sample Prep kit (Illumina, USA) and sequenced on an Illumina HiSeq 4000 in paired-end 150-bp read mode. The data were analyzed free online with the Majorbio I-Sanger Cloud Platform (www.i-sanger.com).

\section{Acknowledgements}

This work was financially supported by the National Natural Science Foundation of China (NSFC Grant Nos. 31701938, 31930096, and 1902034), Collaborative Innovation Center of BAAFS (KJCX201907-2), Ministry of Agriculture and Rural Affairs of China (Grant No. CARS-25), Beijing Scholar Program (Grant No. BSP026) and Guanxi Bagui Scholar Program (Grant No. 2016A11).

\section{Author contributions}

Y.-P.W., J.-F.W., and Y.X. designed the research; Y.-P.W. and J.-F.W. performed the experiments; S.-W.T., J.Z., Y.R., and M.-Y.L. provided technical assistance; Y.-P. W., J.-F.W., and S.-G.G. analyzed the data; Y.-P.W. and J.-F.W. wrote the manuscript; G.-Y.G., H.-Y.Z., and L.Z. provided help with plant material management and manuscript writing. Y.X. revised the manuscript.

\section{Conflict of interest}

The authors declare no competing interests.

Supplementary information The online version contains supplementary material available at https://doi.org/10.1038/s41438-021-00506-1.

Received: 10 November 2020 Revised: 29 December 2020 Accepted: 3 January 2021

Published online: 01 April 2021

\section{References}

1. Guo, Y. et al. Quantitative trait loci for seed size variation in cucurbits-a review. Front. Plant Sci. 11, 304 (2020).

2. Miao, C. B., Wang, D., He, R. Q., Liu, S. K. \& Zhu, J. K. Mutations in MIR396e and MIR396f increase grain size and modulate shoot architecture in rice. Plant Biotechnol. J. 18, 491-501 (2020).

3. Wang, W. et al. Gene editing of the wheat homologs of TONNEAU1-recruiting motif encoding gene affects grain shape and weight in wheat. Plant J. 100, 251-264 (2019).

4. Baboli, Z. M. \& Kordi, A. A. S. Characteristics and composition of watermelon seed oil and solvent extraction parameters effects. J. Am. Oil Chem. Soc. 87 667-671 (2010).

5. Figueiredo, D. \& Kohler, C. Signaling events regulating seed coat development. Biochem. Soc. Trans. 42, 358-363 (2014).

6. Li, N. \& Li, Y. Signaling pathways of seed size control in plants. Curr. Opin. Plant Biol. 33, 23-32 (2016).

7. Li, N., Xu, R. \& Li, Y. H. Molecular networks of seed size control in plants. Annu. Rev. Plant Biol. 70, 435-463 (2019).

8. Li, S. et al. The plant-specific G protein gamma subunit AGG3 influences organ size and shape in Arabidopsis thaliana. N. Phytol. 194, 690-703 (2012).
9. Chakravorty, D. et al. An atypical heterotrimeric G-protein gamma-subunit is involved in guard cell $\mathrm{K}+$-channel regulation and morphological development in Arabidopsis thaliana. Plant J. 67, 840-851 (2011).

10. Mao, $\mathrm{H}$. et al. Linking differential domain functions of the GS3 protein to natural variation of grain size in rice. Proc. Natl Acad. Sci. USA 107, 19579-19584 (2010)

11. Huang, $X$. et al. Natural variation at the DEP1 locus enhances grain yield in rice. Nat. Genet. 41, 494-497 (2009).

12. Liu, Q. et al. G-protein $\beta \gamma$ subunits determine grain size through interaction with MADS-domain transcription factors in rice. Nat. Commun. 9, 852 (2018).

13. Sun, S. et al. A G-protein pathway determines grain size in rice. Nat. Commun 9, 851 (2018).

14. Xia, $\mathrm{T}$. et al. The ubiquitin receptor DA1 interacts with the E3 ubiquitin ligase DA2 to regulate seed and organ size in Arabidopsis. Plant Cell 25, 3347-3359 (2013).

15. Song, X. J., Huang, W., Shi, M., Zhu, M. Z. \& Lin, H. X. A QTL for rice grain width and weight encodes a previously unknown RING-type E3 ubiquitin ligase. Nat. Genet. 39, 623-630 (2007)

16. Bednarek, J. et al. Down-regulation of the TaGW2 gene by RNA interference results in decreased grain size and weight in wheat. J. Exp. Bot. 63, 5945-5955 (2012).

17. Li, Q. et al. Relationship, evolutionary fate and function of two maize coorthologs of rice GW2 associated with kernel size and weight. BMC Plant Biol. 10 143 (2010).

18. Eloy, N. B. et al. SAMBA, a plant-specific anaphase-promoting complex cyclosome regulator is involved in early development and A-type cyclin stabilization. Proc. Natl Acad. Sci. USA 109, 13853-13858 (2012).

19. Zhang, M. et al. Maternal control of embryogenesis by MPK6 and its upstream MKK4/MKK5 in Arabidopsis. Plant J. 92, 1005-1019 (2017).

20. $\mathrm{Xu}, \mathrm{R}$. et al. Control of grain size and weight by the OsMKKK10-OsMKK4-OsMAPK6 signaling pathway in rice. Mol. Plant 11 860-873 (2018).

21. Segami, S. et al. Overexpression of SRS5 improves grain size of brassinosteroidrelated dwarf mutants in rice (Oryza sativa L.). Breed. Sci. 67, 393-397 (2017).

22. Wang, S. et al. The OsSPL16-GW7 regulatory module determines grain shape and simultaneously improves rice yield and grain quality. Nat. Genet. 47, 949-954 (2015).

23. Li, S. et al. TheOsmiR396c-OsGRF4-OsGIF1 regulatory module determines grain size and yield in rice. Plant Biotechnol. J. 14, 2134-2146 (2016).

24. Zhang, Y. et al. Transcription factors SOD7/NGAL2 and DPA4/NGAL3 act redundantly to regulate seed size by directly repressing $\mathrm{KLU}$ expression in Arabidopsis thaliana. Plant Cell 27, 620-632 (2015).

25. Ge, L. et al. Increasing seed size and quality by manipulating BIG SEEDS1 in legume species. Proc. Natl Acad. Sci. USA 113, 12414-12419 (2016).

26. Jofuku, K. D., Omidyar, P. K., Gee, Z. \& Okamuro, J. K. Control of seed mass and seed yield by the floral homeotic gene APETALA2. Proc. Natl Acad. Sci. USA 102, 3117-3122 (2005).

27. Jiang, W. B. et al. Brassinosteroid regulates seed size and shape in Arabidopsis. Plant Physiol. 162, 1965-1977 (2013).

28. Zuo, J. \& Li, J. Molecular genetic dissection of quantitative trait loci regulating rice grain size. Annu. Rev. Genet. 48, 99-118 (2014).

29. Li, Y. et al. Natural variation in GS5 plays an important role in regulating grain size and yield in rice. Nat. Genet. 43, 1266-1269 (2011).

30. Ishimaru, $K$. et al. Loss of function of the IAA-glucose hydrolase gene TGW6 enhances rice grain weight and increases yield. Nat. Genet. 45, 707-711 (2013).

31. Liu, L. et al. Activation of Big Grain1 significantly improves grain size by regulating auxin transport in rice. Proc. Natl Acad. Sci. USA 112, 11102-11107 (2015).

32. Nambara, E. et al. Abscisic acid and the control of seed dormancy and germination. Seed Sci. Res. 20, 55-67 (2010).

33. Lee, K. H. et al. Activation of glucosidase via stress-induced polymerization rapidly increases active pools of abscisic acid. Cell 126, 1109-1120 (2006).

34. Li, Q. et al. Expression analysis of $\beta$-glucosidase genes that regulate abscisic acid homeostasis during watermelon (Citrullus lanatus) development and under stress conditions. J. Plant Physiol. 169, 78-85 (2012).

35. Wang, Y. et al. Abscisic acid pathway involved in the regulation of watermelon fruit ripening and quality trait evolution. PLOS ONE 12, e0179944 (2017).

36. Duan, P. et al. Natural variation in the promoter of GSE5 contributes to grain size diversity in rice. Mol. Plant 10, 685-694 (2017).

37. Seymour, G. B., Østergaard, L., Chapman, N. H., Knapp, S. \& Martin, C. Fruit development and ripening. Annu. Rev. Plant Biol. 64, 219-241 (2013). 
38. Xu, Z. Y. et al. A vacuolar beta-glucosidase homolog that possesses glucoseconjugated abscisic acid hydrolyzing activity plays an important role in osmotic stress responses in Arabidopsis. Plant Cell 24, 2184-2199 (2012).

39. $\mathrm{Li}, \mathrm{Q}$. et al. The role of $\mathrm{FaBG} 3$ in fruit ripening and $\mathrm{B}$. cinerea fungal infection of strawberry. Plant J. 76, 24-35 (2013).

40. Liang, B. et al. Overexpression of the persimmon abscisic acid $\beta$-glucosidase gene (DkBG1) alters fruit ripening in transgenic tomato. Plant J. 102, 1220-1233 (2020).

41. Chen, K., Wang, Y., Zhang, R., Zhang, H. \& Gao, C. CRISPR/Cas genome editing and precision plant breeding in agriculture. Annu. Rev. Plant Biol. 70, 667-697 (2019).

42. Prothro, J., Sandlin, K., Abdel-Haleem, H., Bachlava, E. \& Mcgregor, C. Main and epistatic quantitative trait loci associated with seed size in watermelon. J. Am Soc. Hortic. Sci. 137, 452-457 (2012).

43. Ren, Y. et al. An integrated genetic map based on four mapping populations and quantitative trait loci associated with economically important traits in watermelon (Citrullus lanatus). BMC Plant Biol. 14, 33 (2014).
44. Kim, K. H. et al. Major quantitative trait loci and putative candidate genes for powdery mildew resistance and fruit-related traits revealed by an intraspecific genetic map for watermelon (Citrullus lanatus var. lanatus). PLoS ONE 10, e0145665 (2015).

45. $\mathrm{Li}, \mathrm{N}$. et al. Fine mapping and discovery of candidate genes for seed size in watermelon by genome survey sequencing. Sci. Rep. 8, 843-854 (2018).

46. van der Knaap, E. \& Ostergaard, L. Shaping a fruit: developmental pathways that impact growth patterns. Semin. Cell Dev. Biol. 79, 27-36 (2018).

47. Wu, S. et al. A common genetic mechanism underlies morphological diversity in fruits and other plant organs. Nat. Commun. 9, 4734 (2018).

48. Xing, H. L. et al. A CRISPR/Cas9 toolkit for multiplex genome editing in plants. BMC Plant Biol. 14, 327 (2014).

49. Ren, Y. et al. A tonoplast sugar transporter underlies a sugar accumulation QTL in watermelon. Plant Physiol. 176, 836-850 (2018).

50. Dai, S. J. et al. The functional analysis of SINCED1 in tomato pollen development. Cell. Mol. Life Sci. 75, 3457-3472 (2018). 